\title{
Association of prealbumin levels with contrast- induced acute kidney injury in elderly patients with elective percutaneous coronary intervention
}

\author{
Zhe-Bin You',* \\ Kai-Yang $\operatorname{Lin}^{2, *}$ \\ Wei-Ping Zheng' \\ Chun-Jin Lin' \\ Fan Lin' \\ Tai-Lin Guo' \\ Peng-Li Zhu' \\ Yan-Song Guo
}

'Department of Geriatric Medicine, Fujian Provincial Hospital, Fujian

Key Laboratory of Geriatrics, Fujian Provincial Center for

Geriatrics, Fujian Medical University, Fuzhou, People's Republic of China; ${ }^{2}$ Department of Cardiology, Fujian Provincial Hospital, Fujian Cardiovascular Institute, Fujian Medical University, Fuzhou, People's Republic of China

*These authors contributed equally to this work

Correspondence: Peng-Li Zhu

Department of Geriatric Medicine, Fujian Provincial Hospital, Fujian Key Laboratory of Geriatrics, Fujian Provincial Center for Geriatrics, Fujian Medical University, Fuzhou 35000I, People's Republic of China

Tel +8659 I 882I 7080

Fax +86 59| 87532356

Email zpI7755@I26.com

Yan-Song Guo

Department of Cardiology, Fujian

Provincial Hospital, Fujian Cardiovascular Institute, Fujian Medical University,

Fuzhou 35000I, People's Republic of China

Tel +8659188217410

Fax +86 59| 87532356

Email ysguol234@।26.com
This article was published in the following Dove Press journal:

Clinical Interventions in Aging

Purpose: Inflammatory factors play a critical role in contrast-induced acute kidney injury (CI-AKI). Prealbumin, a nutritional and inflammatory indicator, is a well-established predictor of short- and long-term outcomes in numerous clinical conditions. The current study investigated the association of pre-procedural prealbumin levels with CI-AKI and long-term outcomes in geriatric patients after elective percutaneous coronary intervention (PCI).

Patients and methods: A total of 558 patients aged $\geq 75$ years, who underwent elective PCI between January 2012 and December 2015, were selected for the current study. Pre-procedural prealbumin levels were measured before PCI. Multivariable logistic regression and Cox proportional hazard regression analyses were performed to identify the independent risk factors for CI-AKI and long-term mortality.

Results: Out of 558 patients, 54 developed CI-AKI. The optimal cutoff value of prealbumin for detecting CI-AKI was $185.5 \mathrm{mg} / \mathrm{L}$ with $62.7 \%$ sensitivity and $70.4 \%$ specificity based on the receiver operating characteristic analysis $(\mathrm{C}$-statistic $=0.710 ; 95 \%$ confidence interval [CI] 0.673-0.751). Multivariable analysis demonstrated that prealbumin $\leq 185.5 \mathrm{mg} / \mathrm{L}$ was significantly associated with CI-AKI (odds ratio [OR] 0.397; 95\% CI 0.195-0.808; $P=0.011$ ). Cox regression analysis demonstrated that prealbumin $\leq 185.5 \mathrm{mg} / \mathrm{L}$ was associated with longterm mortality (adjusted hazard ratio [HR] 0.525 ; 95\% CI $0.289-0.952 ; P=0.034$ ) during the follow-up.

Conclusion: Pre-procedural levels of prealbumin were independently associated with an increased risk of CI-AKI and long-term mortality in elderly patients undergoing elective PCI. Keywords: prealbumin, contrast-induced acute kidney injury, percutaneous coronary intervention, elderly

\section{Plain language summary}

Serum prealbumin is a classic indicator of nutritional status and a well-established predictor of outcomes in numerous clinical conditions. On the other hand, the prognostic impact of the nutritional status on contrast-induced acute kidney injury (CI-AKI) and cardiovascular disease is poorly understood. There is growing interest in the association between prealbumin and CI-AKI. Thus, we explored the association of pre-procedural prealbumin levels with CI-AKI and long-term outcomes in geriatric patients who are vulnerable to malnutrition. In this study, elderly patients, aged $\geq 75$ years, undergoing elective percutaneous coronary intervention (PCI) were enrolled, and pre-procedural levels of prealbumin were tested. We found that the patients with CI-AKI were associated with a high rate of inhospital and long-term mortality. The optimal cutoff point of prealbumin was $185.5 \mathrm{mg} / \mathrm{L}$ for predicting CI-AKI with $62.7 \%$ sensitivity and $70.4 \%$ specificity. Thus, prealbumin was found to be associated with long-term mortality in this population cohort in a median 1.8 years of follow-up. In summary, the current study found 
that pre-procedural prealbumin is a significant and independent predictor of CI-AKI and long-term mortality in elderly patients following elective PCI, and the optimal cutoff for predicting CI-AKI was $185.5 \mathrm{mg} / \mathrm{L}$.

\section{Introduction}

Contrast-induced acute kidney injury (CI-AKI) frequently occurs with the increased use of contrast media (CM). ${ }^{1}$ This has led to increased inhospital mortality, dialysis, and a medical burden. ${ }^{2}$ Thus, identifying high-risk patients and early prophylactic measures are crucial for preventing CI-AKI. ${ }^{3}$ Specifically, elderly patients are at high risk of developing CI-AKI and likely to develop deteriorated renal and cardiac functions as compared to the general population. ${ }^{4,5}$

Previous studies have confirmed that inflammatory factors play a critical role in the process of CI-AKI ${ }^{6,7}$ Serum levels of prealbumin were measured to assess an individual's recent nutritional intake and current nutritional state. Accumulating evidence demonstrated that serum prealbumin levels not only indicate the recent nutritional status but also play a role in regulating acute inflammatory diseases. ${ }^{8,9}$ Thus, it has been firmly established that hypo-prealbumin is a significant independent risk factor for mortality in patients with AKI. ${ }^{10-12}$ However, to the best of our knowledge, studies exploring the correlation of prealbumin with CI-AKI and long-term outcomes in elderly patients, who had undergone percutaneous coronary intervention (PCI), are yet lacking.

In the current prospective observational study, we investigated the association of pre-procedural prealbumin levels with CI-AKI and long-term outcomes in geriatric patients post-elective PCI.

\section{Patients and methods Study design and patient population}

Between January 2012 and December 2015, consecutive elderly patients, aged $\geq 75$ years, with coronary artery disease (CAD) undergoing elective PCI, were selected for this study. The exclusion criteria were as follows: 1) patients who are at high risk of acute coronary syndrome (ACS) (ie, admission $<12 \mathrm{~h}$ after ischemia symptom onset or complicated by cardiogenic shock or acute severe heart failure in STEMI patients; refractory angina or hemodynamic instability in patients with non-ST-elevation ACS [NSTE-ACS]) undergoing primary PCI or emergency PCI ( $\mathrm{n}=90) ; 2)$ end-stage renal disease (estimated glomerular filtration rate $[\mathrm{eGFR}]<15 \mathrm{~mL} /$ $\left.\min / 1.73 \mathrm{~m}^{2} ; \mathrm{n}=1\right) ; 3$ ) history of radio-contrast agents $48 \mathrm{~h}$ prior to procedure or $72 \mathrm{~h}$ post-procedure $(\mathrm{n}=3) ; 4$ ) lack of data on pre-procedural or post-procedural serum creatinine ( $\mathrm{SCr}$ ) levels ( $\mathrm{n}=60)$; 5) lack of data on pre-procedural prealbumin levels $(\mathrm{n}=24)$; 6) administration of nonsteroidal antiinflammatory drugs (NSAIDs), aminoglycosides, cisplatin, or other nephrotoxic drugs $48 \mathrm{~h}$ before and $72 \mathrm{~h}$ post-PCI $(n=0)$. Consequently, 558 patients were eligible for recruitment in the study. The protocol fulfilled the requirements of the Declaration of Helsinki and was approved by the ethics committee of the Fujian Provincial Hospital, China (ethics approval number: K2012-001-01). Informed written consents were obtained from all patients after providing them a detailed written description of the potential benefits and risks associated with the study.

\section{$\mathrm{PCl}$}

PCI was performed according to the optimal clinical practice using standard guide catheters, guidewires, balloon catheters, and stents via the femoral or radial approach. The contrast dose was determined at the discretion of the interventional cardiologist. All patients received nonionic and low-osmolarity contrast agents (either iopamiron or ultravist, both $370 \mathrm{mgI} / \mathrm{mL}$ ). In addition, all patients received intravenous isotonic saline $(0.9 \%)$ at a rate of $1 \mathrm{~mL} / \mathrm{kg} / \mathrm{h}$ for $12 \mathrm{~h}$ before and until $24 \mathrm{~h}$ after the procedure (or $0.5 \mathrm{~mL} / \mathrm{kg} / \mathrm{h}$ for $12 \mathrm{~h}$ if the patients were experiencing overt heart failure) according to the guidelines. The patients were treated according to American Heart Association/American College of Cardiology Foundation (AHA/ACCF) guidelines. At the time of admission, serum prealbumin levels were determined by turbidimetric immunoassays using the COBAS 8000 automatic biochemical analyzer (Roche Diagnostics, Basel, Switezerland; prior to PCI), SCr levels were measured at admission and daily for 2 days after contrast administration, and biochemical parameters, including serum albumin, glucose, uric acid, lipid profiles, high-sensitivity C-reactive protein (HsCRP), hemoglobin, white blood cell and platelet counts, and glycated hemoglobin, were also evaluated.

\section{Data collection and end points}

All clinical, laboratory, and angiographic data obtained by reviewing the medical records were assessed and quantified by an independent study team consisting of experienced cardiologists. Follow-up data were obtained during an outpatient clinic visit or by phone. The median follow-up period was 1.8 years. The primary end point was the development of CI-AKI, defined as an absolute increase in $\geq 0.3 \mathrm{mg} / \mathrm{dL}$ or $\geq 50 \%$ from the baseline SCr levels within $48 \mathrm{~h}$ after exposure to $\mathrm{CM}$. Advanced congestive heart failure (CHF) was defined as a New York Heart Association (NYHA) class $>2$, or Killip class $>1$. Anemia was defined as hematocrit $(\mathrm{HCT})<0.39$ (for males) or $<0.36$ (for females). Peri-hypotension was defined as systolic blood pressure 
(SBP) $<80 \mathrm{mmHg}$ for at least $1 \mathrm{~h}$ requiring inotropic support with medications or intra-aortic balloon pump (IABP) within 24 h periprocedurally. ${ }^{13}$ Additional end points were all-cause mortality during the follow-up.

\section{Statistical analyses}

The baseline characteristics were compared between the CIAKI and non-CI-AKI groups. Normally distributed continuous variables were expressed as mean and SD and analyzed using Student's $t$-tests. Non-normally distributed variables were expressed as median and analyzed using Wilcoxon rank-sum test. The categorical variables were represented as percentages and analyzed using chi-square test or Fisher's exact test. The receiver operating characteristic (ROC) curve analysis determined the optimal cutoff value of prealbumin levels to detect CI-AKI using the MedCalc statistical software (MedCalc Software, version 11.4.2.0, Ostend, Belgium). Univariate and multivariate logistic regressions were performed to calculate the odds ratios (ORs) for risk factors of CI-AKI. The variables that were found to be significant in the univariate analysis $(P<0.05)$ and those that were confirmed to be significant in clinical practice were included in the multiple logistic regression analysis. The association of prealbumin with long-term mortality was investigated using
Cox regression analysis. The Kaplan-Meier curve assessed the survival among the four groups that were categorized based on the prealbumin quartiles with log-rank test and between the CI-AKI and non-CI-AKI groups. $P<0.05$ was considered as statistically significant. All statistical analyses were performed using SPSS version 20.0 (IBM Corporation, Armonk, NY, USA).

\section{Results \\ Baseline clinical characteristics and inhospital mortality}

A total of 558 elderly patients were enrolled in this study. The average age of the cohort was $78.82 \pm 3.26$ years, and the cohort was composed of $158(28.3 \%)$ females. The baseline levels of SCr were $0.98 \pm 0.29 \mathrm{mg} / \mathrm{dL}$. Of the total patients selected, 206 (36.9\%) had diabetes.

Table 1 summarizes the univariate analysis of the baseline and procedural characteristics, including the conventional risk factors for CI-AKI between the patients with and without CIAKI. Patients who developed CI-AKI were likely to be older, as well as have CHF, low left ventricular ejection fraction (LVEF) and hemoglobin values, and high eGFR, N-terminal pro-brain-type natriuretic peptide (NT-proBNP), and HsCRP values. These patients presented prolonged procedural

Table I Baseline clinical features in patients with and without $\mathrm{Cl}-\mathrm{AKI}$

\begin{tabular}{|c|c|c|c|c|}
\hline Variables & $\begin{array}{l}\text { Total } \\
(n=558)\end{array}$ & $\begin{array}{l}\text { Cl-AKI (-) } \\
(n=507)\end{array}$ & $\begin{array}{l}\text { CI-AKI (+) } \\
(n=5 I)\end{array}$ & $P$-value \\
\hline \multicolumn{5}{|l|}{ Demographics } \\
\hline Age (years) & $78.82 \pm 3.26$ & $78.72 \pm 3.16$ & $79.84 \pm 4.00$ & 0.02 \\
\hline Sex, female, n (\%) & $158(28.3)$ & $140(27.6)$ & $18(35.3)$ & 0.25 \\
\hline BMI $\left(\mathrm{kg} / \mathrm{m}^{2}\right)$ & $23.61 \pm 3.32$ & $23.60 \pm 3.27$ & $23.77 \pm 3.93$ & 0.79 \\
\hline SBP $(\mathrm{mmHg})$ & | $38.28 \pm 20.57$ & $|38.5| \pm 20.68$ & $135.96 \pm 19.54$ & 0.41 \\
\hline Diastolic blood pressure $(\mathrm{mmHg})$ & $73.27 \pm 25.63$ & $73.22 \pm 26.60$ & $73.83 \pm 12.64$ & 0.87 \\
\hline \multicolumn{5}{|l|}{ Medical history } \\
\hline Smoker, n (\%) & $195(35.3)$ & $178(35.5)$ & $17(33.3)$ & 0.76 \\
\hline Prior PCl, n (\%) & I33 (23.8) & $126(24.9)$ & 7 (I3.7) & 0.08 \\
\hline Prior CABG, n (\%) & $4(0.7)$ & $3(0.6)$ & $\mathrm{I}(2.0)$ & 0.32 \\
\hline Prior MI, n (\%) & $100(17.9)$ & $88(17.4)$ & $12(23.5)$ & 0.27 \\
\hline Hypertension, n (\%) & $436(78.1)$ & $397(78.3)$ & $39(76.5)$ & 0.76 \\
\hline Diabetes, n (\%) & $206(36.9)$ & $187(36.9)$ & $19(37.3)$ & 0.96 \\
\hline Anemia, n (\%) & $26 \mid(46.8)$ & $233(46.0)$ & $28(54.9)$ & 0.22 \\
\hline CHF, n (\%) & $145(26.0)$ & $122(24.1)$ & $23(45.1)$ & $<0.001$ \\
\hline \multicolumn{5}{|l|}{ Laboratory measurements } \\
\hline Prealbumin (g/L) & $202.98 \pm 48.72$ & $206.53 \pm 46.97$ & $\mid 67.61 \pm 52.08$ & $<0.001$ \\
\hline IgNT-proBNP & $2.68 \pm 0.66$ & $2.63 \pm 0.64$ & $3.21 \pm 0.64$ & $<0.001$ \\
\hline Creatinine (mg/dL) & $0.98 \pm 0.29$ & $0.99 \pm 0.29$ & $0.92 \pm 0.32$ & 0.11 \\
\hline $\mathrm{SCr}>1.5 \mathrm{mg} / \mathrm{dL}, \mathrm{n}(\%)$ & $30(5.4)$ & $28(5.5)$ & $2(3.9)$ & 0.63 \\
\hline eGFR & $78.75 \pm 24.02$ & $77.84 \pm 21.58$ & $87.76 \pm 40.30$ & $<0.001$ \\
\hline eGFR class & & & & 0.86 \\
\hline I5-29, n (\%) & $3(0.5)$ & $3(0.6)$ & $0(0)$ & \\
\hline $30-59, \mathrm{n}(\%)$ & $107(19.2)$ & $99(19.5)$ & $8(I 5.7)$ & \\
\hline
\end{tabular}

(Continued) 
Table I (Continued)

\begin{tabular}{|c|c|c|c|c|}
\hline Variables & $\begin{array}{l}\text { Total } \\
(n=558)\end{array}$ & $\begin{array}{l}\text { CI-AKI (-) } \\
(n=507)\end{array}$ & $\begin{array}{l}\text { Cl-AKI (+) } \\
(n=5 I)\end{array}$ & $P$-value \\
\hline $60-89, \mathrm{n}(\%)$ & $291(52.2)$ & $263(5 I .9)$ & $28(54.9)$ & \\
\hline$\geq 90, \mathrm{n}(\%)$ & $157(28.1)$ & $142(28.0)$ & $15(29.4)$ & \\
\hline Uric acid, $\mu \mathrm{mol} / \mathrm{L}$ & $368.50 \pm 105.16$ & $369.68 \pm 105.07$ & $356.86 \pm 106.41$ & 0.41 \\
\hline WBC, $10^{9} / \mathrm{L}$ & $7.01 \pm 2.08$ & $6.94 \pm 1.97$ & $7.66 \pm 2.88$ & 0.02 \\
\hline Hemoglobin $(g / L)$ & $129.19 \pm 16.70$ & $129.88 \pm 16.17$ & $122.25 \pm 20.24$ & $<0.001$ \\
\hline HCT & $0.53 \pm 2.55$ & $0.55 \pm 2.68$ & $0.36 \pm 0.06$ & 0.61 \\
\hline Cholesterol (mmol/L) & $4.13 \pm 1.13$ & $4.15 \pm 1.16$ & $3.95 \pm 0.81$ & 0.23 \\
\hline Triglyceride (mmol/L) & $1.43 \pm 0.89$ & $1.45 \pm 0.91$ & $1.24 \pm 0.54$ & 0.10 \\
\hline High-density lipoprotein (mmol/L) & $1.09 \pm 0.31$ & $1.10 \pm 0.32$ & $1.04 \pm 0.26$ & 0.19 \\
\hline Low-density lipoprotein (mmol/L) & $2.59 \pm 0.97$ & $2.60 \pm 0.99$ & $2.52 \pm 0.72$ & 0.56 \\
\hline Glucose (mmol/L) & $7.11 \pm 3.12$ & $7.11 \pm 3.16$ & $7.09 \pm 2.75$ & 0.98 \\
\hline $\mathrm{HbAlc}(\%)$ & $6.65 \pm 1.22$ & $6.65 \pm 1.23$ & $6.58 \pm 1.11$ & 0.72 \\
\hline LVEF (\%) & $57.37 \pm 7.10$ & $58.02 \pm 6.67$ & $51.05 \pm 8.12$ & $<0.001$ \\
\hline $\mathrm{Hs}-\mathrm{CRP}(\mathrm{mg} / \mathrm{L})$ & $11.61 \pm 22.63$ & $9.63 \pm 17.47$ & $24.38 \pm 41.68$ & 0.01 \\
\hline \multicolumn{5}{|l|}{ Medication } \\
\hline Antiplatelet, n (\%) & $547(98.0)$ & $498(98.2)$ & $49(96.1)$ & 0.29 \\
\hline Statin, n (\%) & $544(97.5)$ & $495(97.6)$ & $49(96.1)$ & 0.50 \\
\hline$\beta$-blocker, n (\%) & $451(80.8)$ & $412(81.3)$ & $39(76.5)$ & 0.41 \\
\hline ACEI/ARB, n (\%) & $460(82.4)$ & $418(82.4)$ & $42(82.4)$ & 0.99 \\
\hline Diuretics, n (\%) & $221(39.6)$ & 187 (36.9) & $34(66.7)$ & $<0.001$ \\
\hline \multicolumn{5}{|l|}{ Procedure performed } \\
\hline Number of diseased vessels (n) & $2.39 \pm 0.81$ & $2.38 \pm 0.82$ & $2.47 \pm 0.73$ & 0.47 \\
\hline \multicolumn{5}{|l|}{ Vessel disease } \\
\hline LM & $55(9.9)$ & $49(9.7)$ & $6(11.8)$ & 0.63 \\
\hline LAD & $500(89.6)$ & $452(89.2)$ & $48(94.1)$ & 0.27 \\
\hline LCX & $369(66.1)$ & $336(66.3)$ & $33(64.7)$ & 0.82 \\
\hline $\mathrm{RCA}$ & $391(70.1)$ & $354(69.8)$ & $37(72.5)$ & 0.69 \\
\hline Stent length & $42.64 \pm 23.29$ & $42.11 \pm 23.18$ & $47.87 \pm 23.99$ & 0.11 \\
\hline Perioperative hypotension, n (\%) & $10(1.8)$ & $7(1.4)$ & $3(5.9)$ & 0.06 \\
\hline Contrast volume & $222.67 \pm 59.78$ & $223.40 \pm 59.64$ & $215.42 \pm 61.26$ & 0.38 \\
\hline Contrast volume $>$ I50 mL, n (\%) & $480(9 \mid .4)$ & $439(92.0)$ & $4 \mathrm{I}(85.4)$ & 0.12 \\
\hline
\end{tabular}

Abbreviations: ACEI/ARB, angiotensin-converting enzyme inhibitor/angiotensin receptor blocker; BMI, body mass index; CABG, coronary artery bypass grafting; $\mathrm{CHF}$, congestive heart failure; $\mathrm{Cl}-\mathrm{AKI}$, contrast-induced acute kidney injury; eGFR, estimated glomerular filtration rate; HbAIc, glycated hemoglobin; HCT, hematocrit; Hs-CRP, high-sensitivity C-reactive protein; LAD, left anterior descendant; LCX, left circular branch; LM, left main stem; LVEF, left ventricular ejection fraction; MI, myocardial infarction; NT-proBNP, N-terminal pro-brain-type natriuretic peptide; PCl, percutaneous coronary intervention; RCA, right coronary artery; SBP, systolic blood pressure; $\mathrm{SCr}$, serum creatinine; WBC, white blood cells.

durations or multivessel disease and were frequently treated with diuretics. Furthermore, these patients with CI-AKI were likely to have low prealbumin and albumin levels.

Moreover, patients with CI-AKI exhibited a significantly higher rate of inhospital mortality $(5.90 \%$ vs $0.40 \%$, $P<0.0001$ ) as compared to those without CI-AKI (Figure 1).

\section{ROC analysis and multivariable factors for predicting $\mathrm{Cl}-\mathrm{AKI}$}

Individuals with low pre-procedural prealbumin concentrations were likely to develop CI-AKI (quartile (Q) Q1, Q2, Q3, Q4: 19.3\%, 9.4\%, 4.3\%, 3.6\%, respectively; $P<0.0001$; Figure 2).

ROC analysis indicated that a cutoff value of $185.5 \mathrm{mg} / \mathrm{L}$ for prealbumin levels could predict CI-AKI with a sensitivity of $62.7 \%$ and a specificity of $70.4 \%$ (C-statistic $=0.710 ; 95 \%$ confidence interval [CI] 0.673-0.751; Figure 3).

Univariate logistic regression determined that age, hemoglobin, lgNT-proBNP, CHF, acute myocardial infarction,

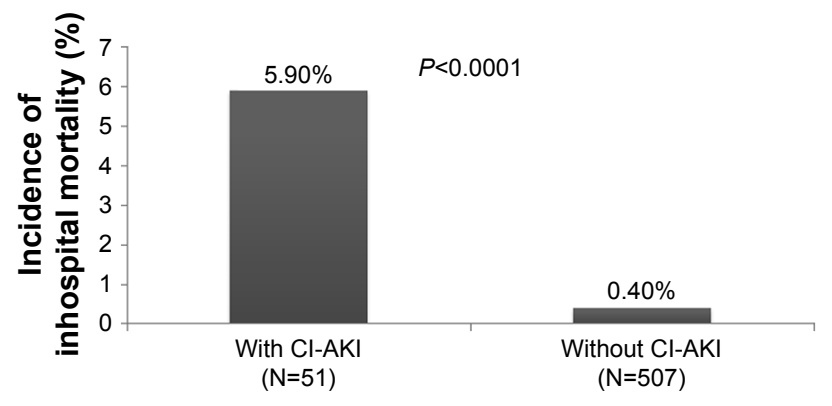

Figure I Inhospital mortality between patients with and without $\mathrm{Cl}-\mathrm{AKI}$. Abbreviation: $\mathrm{Cl}-\mathrm{AKI}$, contrast-induced acute kidney injury. 


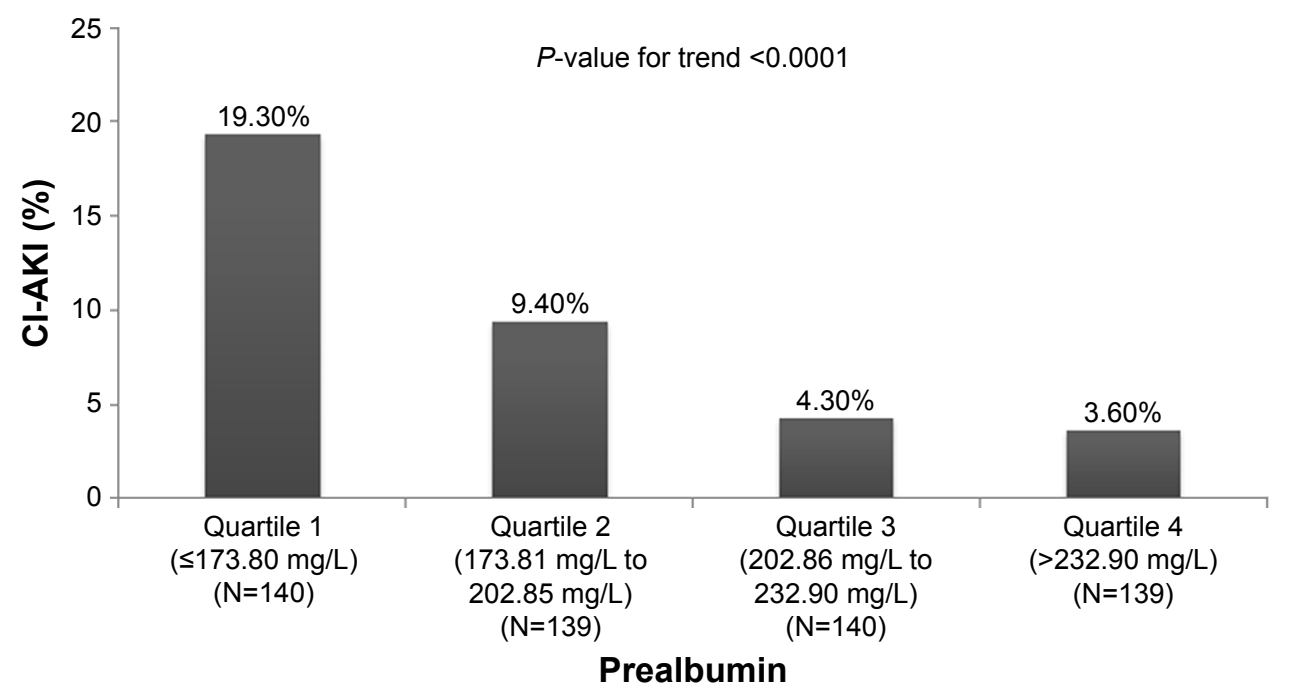

Figure 2 Relationship between prealbumin levels and patients developing $\mathrm{CI}-\mathrm{AKI}$. Abbreviation: $\mathrm{Cl}-\mathrm{AKI}$, contrast-induced acute kidney injury.

prealbumin $\leq 185.5 \mathrm{mg} / \mathrm{L}$, and peri-hypotension were associated with CI-AKI (all $P<0.05$ ). The three common risk factors used in clinical practice $(\mathrm{SCr}>1.5 \mathrm{mg} / \mathrm{dL}$, diabetes, and contrast volume $>150 \mathrm{~mL}$ ) were included in the multiple logistic regression analysis. Multivariate analysis indicated that prealbumin levels (OR 0.397; 95\% CI $0.195-0.808 ; P=0.011$ ), peri-hypotension (OR 5.489; 95\% CI 1.101-27.360; $P=0.038$ ), and lgNT-proBNP (OR 4.643; 95\% CI 2.201-9.791; $P<0.0001)$ remained significant

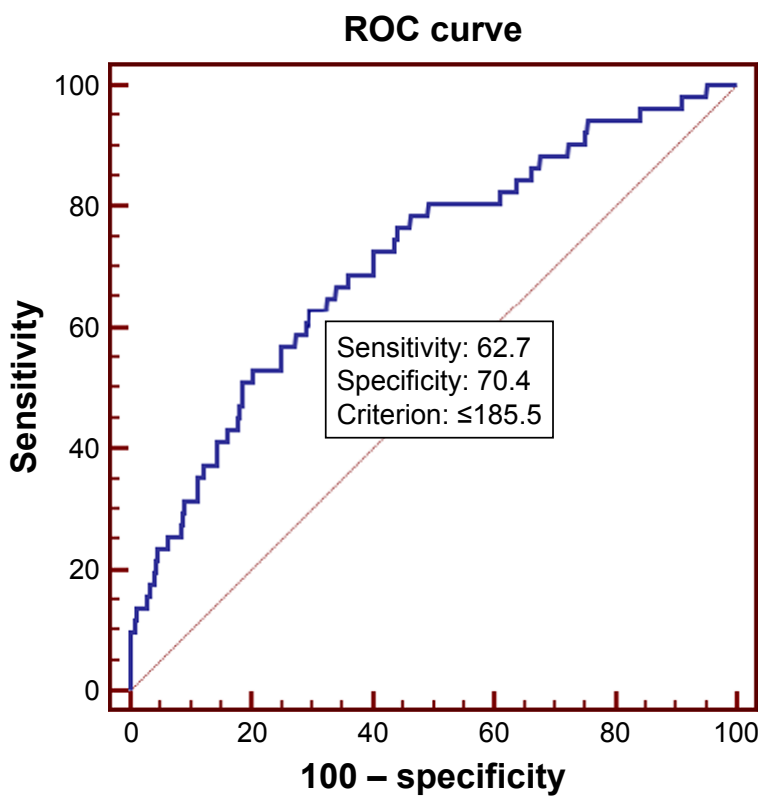

Figure 3 ROC curve analysis.

Notes: ROC curve analysis demonstrated that a prealbumin cutoff value of 185.5 was optimal and exhibited $62.7 \%$ sensitivity and $70.4 \%$ specificity for detecting Cl-AKI. The C-statistic was 0.710 (0.673-0.75I).

Abbreviations: $\mathrm{Cl}-\mathrm{AKI}$, contrast-induced acute kidney injury; $\mathrm{ROC}$, receiver operator characteristic. predictors for the development of CI-AKI in elderly patients after elective PCI (Table 2).

\section{Pre-procedural prealbumin and long-term outcomes}

Kaplan-Meier curve demonstrated that low levels of prealbumin presented high all-cause mortality based on the cutoff value of prealbumin $(185.5 \mathrm{mg} / \mathrm{L})$ and four quartiles of prealbumin levels ( $P=0.0006, P=0.0008$; Figures 4 and 5). Cox regression analysis showed that pre-procedural prealbumin levels were an independent risk factor for long-term mortality (hazard ratio [HR] 0.525 ; 95\% CI 0.289-0.952; $P=0.034$ ) after adjusting for other risk factors including age, $\mathrm{SCr}>1.5 \mathrm{mg} / \mathrm{dL}$, and lgNT-proBNP (Table 3). Patients who developed CI-AKI faced a high rate of all-cause mortality as compared to those without CI-AKI $(P<0.0001$; Figure 6).

\section{Discussion}

The current study demonstrated that elevated levels of preprocedural prealbumin were associated with an increased risk of developing CI-AKI and poor inhospital and longterm outcomes in elderly patients undergoing elective PCI. After adjusting for the confounding factors, levels of pre-procedural prealbumin $\leq 185.50 \mathrm{mg} / \mathrm{L}$ were the optimal cutoff value (based on ROC) for predicting CI-AKI and long-term mortality.

CI-AKI is one of the most common reasons for hospitalacquired kidney injury and is known to rise the morbidity and mortality, increase health care costs, as well as, prolong the duration of hospitalization. ${ }^{14,15}$ Meta-analysis by Song et $\mathrm{al}^{16}$ showed that the risk of developing CI-AKI in the 
Table 2 Univariate and multivariate logistic regression analyses for $\mathrm{Cl}-\mathrm{AKI}$

\begin{tabular}{|c|c|c|c|c|c|c|}
\hline \multirow[t]{2}{*}{ Risk factors } & \multicolumn{3}{|c|}{ Univariate logistic regression } & \multicolumn{3}{|c|}{ Multivariate logistic regression } \\
\hline & OR & $95 \% \mathrm{Cl}$ & $P$-value & OR & $95 \% \mathrm{Cl}$ & $P$-value \\
\hline Age & 1.098 & $1.015-1.188$ & 0.020 & 1.086 & $0.992-1.188$ & 0.075 \\
\hline Hemoglobin & 0.974 & $0.958-0.991$ & 0.002 & 0.989 & $0.969-1.008$ & 0.288 \\
\hline IgNT-proBNP & 4.421 & $2.584-7.564$ & $<0.0001$ & 4.643 & $2.201-9.791$ & $<0.000$ I \\
\hline $\mathrm{SCr}>1.5 \mathrm{mg} / \mathrm{dL}$ & 0.698 & $0.161-3.020$ & 0.631 & 0.476 & $0.098-2.315$ & 0.358 \\
\hline $\mathrm{CHF}$ & 2.592 & $1.440-4.667$ & 0.001 & 1.323 & $0.607-2.885$ & $0.48 \mathrm{I}$ \\
\hline Prealbumin $>185.5 \mathrm{mg} / \mathrm{L}$ & $0.27 \mid$ & $0.150-0.491$ & $<0.0001$ & 0.397 & $0.195-0.808$ & 0.011 \\
\hline Peri-hypotension & 4.465 & I.II8-17.826 & 0.034 & 5.489 & I.10I-27.360 & 0.038 \\
\hline Acute $\mathrm{MI}$ & 2.920 & $1.630-5.232$ & $<0.0001$ & 0.522 & $0.214-1.273$ & 0.153 \\
\hline Diabetes & 1.016 & $0.560-1.843$ & 0.958 & 0.961 & $0.477-1.939$ & 0.912 \\
\hline Contrast volume $>150 \mathrm{~mL}$ & 0.407 & $0.131-1.26 \mid$ & 0.119 & 0.550 & $0.201-1.500$ & 0.358 \\
\hline
\end{tabular}

Abbreviations: $\mathrm{CHF}$, congestive heart failure; $\mathrm{Cl}$, confidence interval; $\mathrm{Cl}$-AKI, contrast-induced acute kidney injury; MI, myocardial infarction; NT-proBNP, N-terminal pro-brain-type natriuretic peptide; $\mathrm{OR}$, odds ratio; $\mathrm{SCr}$, serum creatinine.

elderly was twofold more than that in younger patients even after adjustment for other risk factors. The estimated overall incidence of CI-AKI following intravascular CM administration was $13.6 \%$ in the elderly, which is higher than the previously reported incidence in a nonsegregated population. A study in Japan ${ }^{5}$ concluded that elderly patients had a higher incidence of CI-AKI (14\%) than those in the control group (9\%). Furthermore, CI-AKI in elderly patients was shown to be associated with prolonged renal dysfunction and poor prognosis. In the current study, we found that the incidence of CI-AKI was $10 \%$, and patients with CI-AKI were strongly associated with higher risk of death and mildly increased incidence of inhospital events. The incidence of CI-AKI in our study cohort was lower than that reported previously. This discrepancy may be explained by excluding the patients undergoing primary PCI. Nevertheless, it is vital to identify the elderly patients with high risk of CI-AKI.

Prealbumin, also known as transthyretin, is a globular protein synthesized by parenchymal cells. It forms a complex

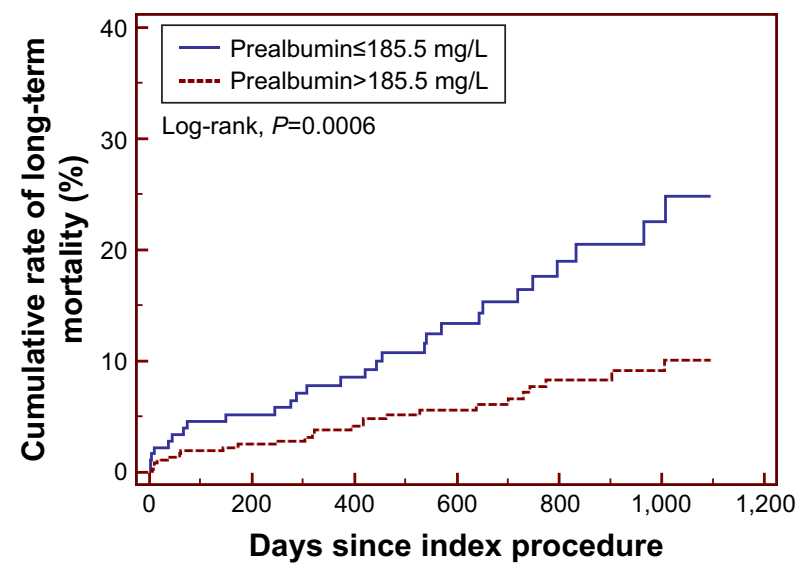

Figure 4 Kaplan-Meier curves demonstrate the cumulative mortality for patients based on the cutoff value of prealbumin levels (185.5 mg/L). with retinol-binding protein and transports up to $90 \%-95 \%$ of retinol/vitamin A and $20 \%$ of circulating thyroid hormones in the body. ${ }^{17}$ Prealbumin, like albumin, is not only a nutrition screening and assessment tool but also a part of the group of negative acute-phase reactants that decrease in the presence of acute inflammatory diseases. ${ }^{8}$ Several studies have demonstrated that prealbumin levels constitute a wellestablished predictor of short- and long-term outcomes in numerous clinical conditions, including heart failure, ${ }^{18,19}$ cerebral infarction, ${ }^{20}$ and hemodialysis. ${ }^{21}$ Recent studies have suggested that prealbumin level is a valuable biomarker for assessing prognosis in patients with AKI. Perez et al ${ }^{10}$ showed that serum prealbumin levels $<11 \mathrm{mg} / \mathrm{dL}$ were an independent predictor of inhospital mortality as assessed by multivariate analyses (HR 2.10; 95\% CI 1.08-4.08), and inhospital mortality of AKI patients decreased by $29 \%$ for every $5 \mathrm{mg} / \mathrm{dL}$ increase in serum prealbumin levels (HR 0.71; $95 \%$ CI $0.52-0.96$ ). Wang et a ${ }^{12}$ demonstrated that serum prealbumin levels were superior to serum albumin levels in

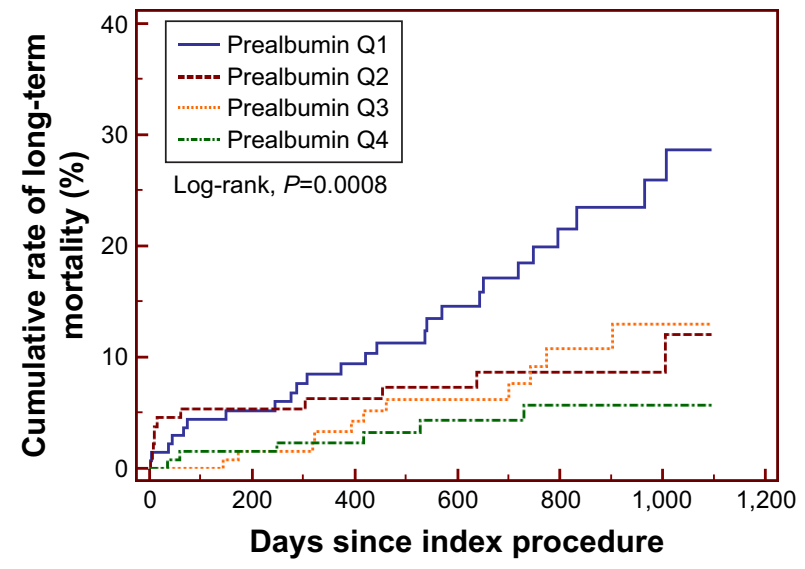

Figure 5 Kaplan-Meier curves demonstrate the cumulative mortality for patients based on prealbumin quartile $(\mathrm{Q})$ levels. 
Table 3 Univariate and multivariate Cox analyses: independent risk factors for long-term mortality

\begin{tabular}{|c|c|c|c|c|c|c|}
\hline \multirow[t]{2}{*}{ Risk factors } & \multicolumn{3}{|c|}{ Univariate } & \multicolumn{3}{|c|}{ Multivariate } \\
\hline & HR & $95 \% \mathrm{Cl}$ & $P$-value & HR & $95 \% \mathrm{Cl}$ & $P$-value \\
\hline Age & 1.086 & $1.006-1.173$ & 0.034 & 1.065 & $0.985-1.150$ & 0.113 \\
\hline IgNT-proBNP & 2.159 & $1.410-3.306$ & 0.000 & 1.674 & $1.025-2.733$ & 0.039 \\
\hline $\mathrm{SCr}>1.5 \mathrm{mg} / \mathrm{dL}$ & 2.771 & $1.25 \mathrm{I}-6.137$ & 0.012 & 2.973 & $1.305-6.776$ & 0.010 \\
\hline $\mathrm{CHF}$ & 1.622 & $0.930-2.829$ & 0.088 & 0.972 & $0.522-1.809$ & 0.928 \\
\hline Prealbumin $>185.5 \mathrm{mg} / \mathrm{L}$ & 0.401 & $0.234-0.689$ & 0.001 & 0.525 & $0.289-0.952$ & 0.034 \\
\hline Peri-hypotension & 2.182 & $0.933-5.107$ & 0.072 & $1.79 \mid$ & $0.75|-4.27|$ & 0.189 \\
\hline
\end{tabular}

Abbreviations: $\mathrm{CHF}$, congestive heart failure; $\mathrm{Cl}$, confidence interval; $\mathrm{HR}$, hazard ratio; NT-proBNP, N-terminal pro-brain-type natriuretic peptide; SCr, serum creatinine.

predicting mortality in AKI after adjusting for multivariables. Moreover, the current study showed that low prealbumin levels were strongly associated with long-term mortality in elderly patients undergoing elective PCI after adjusting for the associated risk factors (HR 0.525 ; 95\% CI 0.289-0.952; $P=0.034$ ), which was in agreement with that from previous studies. However, to our knowledge, a study that investigated the relationship between the pre-procedural prealbumin levels and CI-AKI, as well as long-term mortality in patients who underwent elective PCI, is yet lacking.

Recently, the predictive value of albumin in AKI was highlighted. A meta-analysis by Wiedermann et a ${ }^{22}$ provided evidence that low levels of serum albumin acted as an independent predictor of AKI and death following AKI. Moreover, a prospective, single-center, randomized, parallelarm, double-blind trial conducted by Lee et $\mathrm{al}^{23}$ evaluated the effects of administration of $20 \%$ human albumin solution vs saline on the incidence of postoperative AKI in adult patients with hypoalbuminemia $(<4.0 \mathrm{~g} / \mathrm{dL})$, who were undergoing off-pump coronary artery bypass surgery. Multivariate logistic regression revealed a protective effect of albumin therapy on the renal function (OR $0.42 ; 95 \%$ CI $0.21-0.83 ; P=0.012$ ).

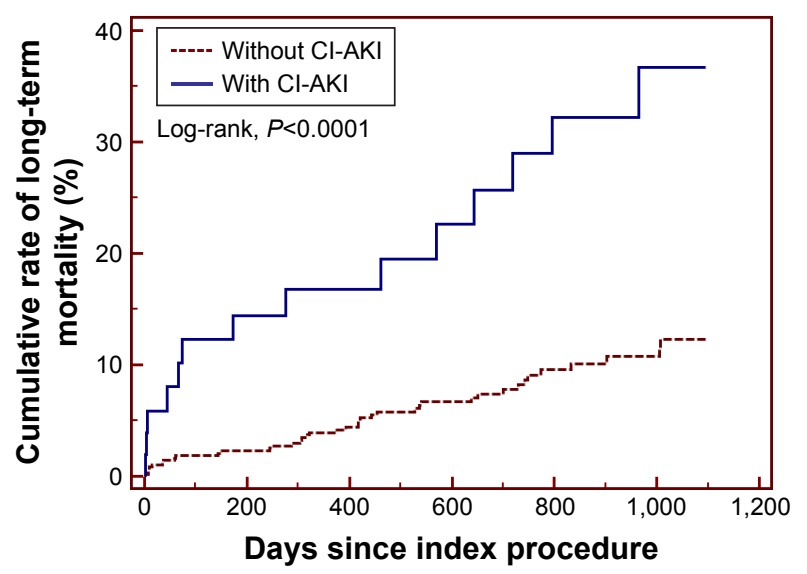

Figure 6 Kaplan-Meier curves demonstrate the cumulative mortality for patients with and without $\mathrm{Cl}-\mathrm{AKI}$.

Abbreviation: $\mathrm{Cl}-\mathrm{AKI}$, contrast-induced acute kidney injury.
A recent study by Murat et $\mathrm{al}^{24}$ retrospectively assessed the impact of serum albumin levels on CI-AKI occurrence in a cohort of 890 patients with ACS treated with PCI. The study demonstrated that serum albumin was inversely associated with AKI risk and independently predicted the occurrence of AKI along with a number of other variables. Inflammation, endothelial dysfunction, and oxidative stress are speculated to be major mechanisms involved in the development of CI-AKI. With a short half-life, a high rate of hepatic synthesis, and predictable catabolic rate, prealbumin is a more sensitive indicator of systemic inflammation and better marker for evaluating nutritional status as compared to albumin. The current study revealed that low prealbumin levels were independently associated with increased CI-AKI and long-term mortality and confirmed the hypothesis that prealbumin might be a potential surrogate to predict CI-AKI and long-term outcomes.

The reasons for the strong association between low preprocedural prealbumin levels and high risk of CI-AKI are not yet clear; however, the potential mechanisms underlying the association of low prealbumin concentrations with CI-AKI may include the following. One possible underlying mechanism linking CI-AKI and prealbumin levels might be malnutrition predisposing the patients to CI-AKI. Malnutrition manifests as weight loss associated with protective metabolic responses such as decreased basic metabolic rates and preservation of lean body mass at the expense of fat mass. ${ }^{25}$ Adipose tissue has been shown to increase the expression of soluble TNF- $\alpha$ receptors, which might play a role in the neutralization of the biological effects of TNF- $\alpha,{ }^{26}$ thereby providing a protective advantage for well-nourished patients. Obese patients also have high levels of circulating lipoproteins that might bind and detoxify the lipopolysaccharides responsible for the release of systematic inflammatory cytokines. ${ }^{27}$ In addition, adiponectin, one of the adipokines secreted by adipose tissue, may exert a protective role in renal function by improving endothelial dysfunction, reducing 
oxidative stress, and upregulating the expression of endothelial nitric oxide synthase (eNOS) via the activation of AMPK by AdipoR1 and PPAR- $\alpha$ signaling pathway by AdipoR. ${ }^{28,29}$ In addition, loss of muscle mass was associated with decreased stroke volume and cardiac output, which in turn, aggravates renal ischemia in patients with elective PCI. ${ }^{30,31}$

Another possible underlying mechanism may be the increased inflammatory status predisposing the patients to CI-AKI. Inflammation is one of the dominating factors in the pathogenesis of CI-AKI. Multiple inflammatory cytokines including interleukin (IL)-1, IL-6, and TNF- $\alpha$ suppress the synthesis of prealbumin and stimulate the generation of Hs-CRP. ${ }^{32,33}$ High levels of Hs-CRP impair the endothelial function by attenuating the NO production via the downregulation of eNOS mRNA and facilitate apoptosis in endothelial cells, ${ }^{34}$ which leads to vascular impairment, decreased renal blood flow, and exacerbation of renal function. The current study demonstrated that high Hs-CRP levels were associated with an elevated risk of CI-AKI, which was according to the previous findings.

Nevertheless, the current study had several limitations. 1) All patients selected for this study were from a single health care institution, and thus, a selection bias during enrollment was inevitable. 2) The cause of death was not recorded, rendering difficulty in establishing a link between serum prealbumin levels and the cause of death. 3) Urine output measurements are critical, which were not conducted, and hence, the number of patients with CI-AKI may have been underestimated. 4) This study was performed on a relatively small cohort, thereby necessitating further evaluation using large cohorts from multicenter institutions. Despite these limitations, our results provided useful insights into the correlation of serum prealbumin levels with the incidence of CI-AKI.

\section{Conclusion}

The current study demonstrated that $185.5 \mathrm{mg} / \mathrm{L}$ was the optimal cutoff value of pre-procedural prealbumin and an effective predictor of CI-AKI and long-term mortality in elderly patients after elective PCI. These findings could guide use of preventive measures and therapy to alleviate CI-AKI.

\section{Acknowledgments}

We thank the staff of the medical record office at Fujian Provincial Hospital for compiling the medical records of the selected patients in this study. The study was supported by a grant from the Startup Fund for Scientific Research, Fujian Medical University (grant number: 2016QH113).

\section{Disclosure}

The authors report no conflicts of interest in this work.

\section{References}

1. Mehran R, Nikolsky E. Contrast-induced nephropathy: definition, epidemiology, and patients at risk. Kidney Int Suppl. 2006;100:S11-S15.

2. McCullough PA. Contrast-induced acute kidney injury. J Am Coll Cardiol. 2008;51(15):1419-1428.

3. Geenen RW, Kingma HJ, van der Molen AJ. Contrast-induced nephropathy: pharmacology, pathophysiology and prevention. Insights Imaging. 2013;4(6):811-820.

4. Lian D, Liu Y, Liu YH, Li HL, Duan CY, Yu DQ. Pre-procedural risk score of contrast-induced nephropathy in elderly patients undergoing elective coronary angiography. Int Heart J. 2017;58(2):197-204.

5. Motohiro M, Kamihata H, Suwa Y, et al. [Incidence and clinical outcome of contrast-induced nephropathy in the elderly patients]. Japanese Journal of Geriatrics. 2013;50(2):227-232.

6. Seeliger E, Sendeski M, Rihal CS, Persson PB. Contrast-induced kidney injury: mechanisms, risk factors, and prevention. Eur Heart $J$. 2012;33(16):2007-2015.

7. Azzalini L, Spagnoli V, Ly HQ. Contrast-induced nephropathy: from pathophysiology to preventive strategies. Can J Cardiol. 2016;32(2): 247-255.

8. Dennis RA, Johnson LE, Roberson PK, et al. Changes in prealbumin, nutrient intake, and systemic inflammation in elderly recuperative care patients. J Am Geriatr Soc. 2008;56(7):1270-1275.

9. Myron JA, Merlini G, Sheldon J, Ichihara K; Scientific Division Committee on Plasma Proteins (C-PP), International Federation of Clinical Chemistry and Laboratory Medicine (IFCC). Clinical indications for plasma protein assays: transthyretin (prealbumin) in inflammation and malnutrition. Clin Chem Lab Med. 2007;45(3):419-426.

10. Perez VJR, Bes-Rastrollo M, Monedero P, de Irala J, Lavilla FJ. Impact of prealbumin levels on mortality in patients with acute kidney injury: an observational cohort study. J Ren Nutr. 2008;18(3):262-268.

11. Murray PT. Association of prealbumin level with mortality in patients with acute kidney injury. Nat Clin Pract Nephrol. 2008;4(10): 528-529.

12. Wang W, Pan Y, Tang X, et al. Serum prealbumin and its changes over time are associated with mortality in acute kidney injury. Sci Rep. 2017;7:41493.

13. Mehran R, Aymong ED, Nikolsky E, et al. A simple risk score for prediction of contrast-induced nephropathy after percutaneous coronary intervention: development and initial validation. $J$ Am Coll Cardiol. 2004;44(7):1393-1399.

14. Best PJ, Lennon R, Ting HH, et al. The impact of renal insufficiency on clinical outcomes in patients undergoing percutaneous coronary interventions. J Am Coll Cardiol. 2002;39(7):1113-1119.

15. McCullough PA, Adam A, Becker CR, et al; CIN Consensus Working Panel. Epidemiology and prognostic implications of contrast-induced nephropathy. Am J Cardiol. 2006;98(6A):5K-13K.

16. Song W, Zhang T, Pu J, Shen L, He B. Incidence and risk of developing contrast-induced acute kidney injury following intravascular contrast administration in elderly patients. Clin Interv Aging. 2014;9:85-93.

17. Ingenbleek $Y$, De Visscher M, De Nayer P. Measurement of prealbumin as index of protein-calorie malnutrition. Lancet. 1972;2(7768):106-109.

18. Franco J, Formiga F, Trullas JC, et al; RICA Investigators Group. Impact of prealbumin on mortality and hospital readmission in patients with acute heart failure. Eur J Intern Med. 2017;43:36-41.

19. Lourenço P, Silva S, Friões F, et al. Low prealbumin is strongly associated with adverse outcome in heart failure. Heart. 2014;100(22): 1780-1785. 
20. Gao C, Zhang B, Zhang W, Pu S, Yin J, Gao Q. Serum prealbumin (transthyretin) predict good outcome in young patients with cerebral infarction. Clin Exp Med. 2011;11(1):49-54.

21. Chertow GM, Ackert K, Lew NL, Lazarus JM, Lowrie EG. Prealbumin is as important as albumin in the nutritional assessment of hemodialysis patients. Kidney Int. 2000;58(6):2512-2517.

22. Wiedermann CJ, Wiedermann W, Joannidis M. Hypoalbuminemia and acute kidney injury: a meta-analysis of observational clinical studies. Intensive Care Med. 2010;36(10):1657-1665.

23. Lee EH, Kim WJ, Kim JY, et al. Effect of exogenous albumin on the incidence of postoperative acute kidney injury in patients undergoing off-pump coronary artery bypass surgery with a preoperative albumin level of less than $4.0 \mathrm{~g} / \mathrm{dl}$. Anesthesiology. 2016;124(5):1001-1011.

24. Murat SN, Kurtul A, Yarlioglues M. Impact of serum albumin levels on contrast-induced acute kidney injury in patients with acute coronary syndromes treated with percutaneous coronary intervention. Angiology. 2015;66(8):732-737.

25. Mak RH, Cheung W. Energy homeostasis and cachexia in chronic kidney disease. Pediatr Nephrol. 2006;21(12):1807-1814.

26. Mohamed-Ali V, Goodrick S, Bulmer K, Holly JM, Yudkin JS, Coppack SW. Production of soluble tumor necrosis factor receptors by human subcutaneous adipose tissue in vivo. Am J Physiol. 1999; 277(6 pt 1):E971-E975.
27. Niebauer J, Volk HD, Kemp M, et al. Endotoxin and immune activation in chronic heart failure: a prospective cohort study. Lancet. 1999; 353(9167):1838-1842.

28. Ruan H, Dong LQ. Adiponectin signaling and function in insulin target tissues. J Mol Cell Biol. 2016;8(2):101-109.

29. Yamauchi T, Nio Y, Maki T, et al. Targeted disruption of AdipoR1 and AdipoR2 causes abrogation of adiponectin binding and metabolic actions. Nat Med. 2007;13(3):332-339.

30. Carbone S, Lavie CJ, Arena R. Obesity and heart failure: focus on the obesity paradox. Mayo Clin Proc. 2017;92(2):266-279.

31. McCullough PA, Adam A, Becker CR, et al; CIN Consensus Working Panel. Risk prediction of contrast-induced nephropathy. Am J Cardiol. 2006;98(6A):27K-36K.

32. Kalantar-Zadeh K, Anker SD, Horwich TB, Fonarow GC. Nutritional and anti-inflammatory interventions in chronic heart failure. $\mathrm{Am} \mathrm{J}$ Cardiol. 2008;101(11A):89E-103E

33. Beck FK, Rosenthal TC. Prealbumin: a marker for nutritional evaluation. Am Fam Physician. 2002;65(8):1575-1578.

34. Verma S, Wang CH, Li SH, et al. A self-fulfilling prophecy: C-reactive protein attenuates nitric oxide production and inhibits angiogenesis. Circulation. 2002;106(8):913-919.
Clinical Interventions in Aging

\section{Publish your work in this journal}

Clinical Interventions in Aging is an international, peer-reviewed journal focusing on evidence-based reports on the value or lack thereof of treatments intended to prevent or delay the onset of maladaptive correlates of aging in human beings. This journal is indexed on PubMed Central, MedLine,

\section{Dovepress}

CAS, Scopus and the Elsevier Bibliographic databases. The manuscript management system is completely online and includes a very quick and fair peer-review system, which is all easy to use. Visit http://www.dovepress. com/testimonials.php to read real quotes from published authors. 